\title{
Review of the Frontier Workshop and Q-slope results
}

\author{
G. Ciovati \\ Thomas Jefferson National Accelerator Facility (TJNAF), Newport News, VA, USA
}

\begin{abstract}
Over the last few years, significant progress has been made to produce field emission free niobium surfaces. Nowadays, the major limitation towards achieving the critical field in radio-frequency (rf) superconducting cavities made of bulk niobium of high purity is represented by the so-called "high field $Q$-slope" or " $Q$-drop". This phenomenon is characterized by a sharp decrease of the cavity quality factor, in absence of field emission, starting at a peak surface magnetic field of the order of $100 \mathrm{mT}$. It has been observed that these losses are usually reduced by a low-temperature "in-situ" baking, typically at $100-120^{\circ} \mathrm{C}$ for $24-48 \mathrm{~h}$.

Several models have been proposed to explain the high field $Q$-slope and many experiments have been conducted in different laboratories to validate such models. A three-day workshop was held in Argonne in September 2004 to present and discuss experimental and theoretical results on the present limitations of superconducting $\mathrm{rf}$ cavities. In this paper, we will focus on the high field $Q$-slope by reviewing the results presented at the workshop along with other experimental data. In order to explain the $Q$ drop and the baking effect we will discuss an improved version of the oxygen diffusion model.
\end{abstract}


Keywords: surface impedance, niobium, radio-frequency cavities

PACS: $74.25 . \mathrm{Nf}$

\section{Corresponding author:}

Gianluigi Ciovati

TJNAF

12000 Jefferson Avenue

Newport News VA, 23606

USA

Phone: +1 7572695945

Fax: $\quad+17572697658$

E-mail: gciovati@jlab.org 


\section{Introduction}

Over the last decade, a steady improvement of the cleaning procedures and techniques allowed to produce superconducting radio-frequency (rf) cavities made of bulk niobium which could sustain peak surface electric fields $\left(E_{p}\right)$ beyond $30 \mathrm{MV} / \mathrm{m}$, without field emission. A new kind of "anomalous" losses were then discovered [1, 2], starting at peak surface magnetic field $\left(B_{p}\right)$ of the order of $100 \mathrm{mT}\left(E_{p} \approx 40 \mathrm{MV} / \mathrm{m}\right)$, which cause a rapid decrease of the cavity quality factor $\left(Q_{0}\right)$, without X-rays, and therefore limits the maximum gradient the cavity can be operated at in a particle accelerator. This high field losses are often called "high field $Q$-slope" or " $Q$-drop". Although several models have been proposed to explain the origin of such losses, none of them gives a complete description of all the experimental results. In spite of this lack of understanding, it was found that a low-temperature $\left(100-140{ }^{\circ} \mathrm{C}\right)$ "in-situ" baking of the cavity for about $48 \mathrm{~h}$ allows to reduce the $Q$-drop $[3,4]$ and this procedure is now commonly used as the final step in the preparation of a superconducting niobium cavity. Nevertheless, understanding the origin of the $Q$-drop and the baking effect is a valuable challenge both from the scientific point of view and to reliably produce cavities achieving the superconducting critical field. In this spirit, a "Workshop on Pushing the Limits of RF Superconductivity" was organized by Argonne National Laboratory in September 2004 to review experimental and theoretical results and to discuss how to further improve the technology of rf superconductivity applied to cavities for particle accelerators. The workshop covered a wide range of topics such as ultimate field limits, new materials, new geometries, high $Q$, field emission, $Q$-slopes and future research paths. In this contribution we will focus on the high field $Q$-drop, by reviewing the large amount of 
experimental data accumulated over the last seven years both on cavities and samples. Differences in the onset field of the $Q$-drop and in the baking effect appear for cavities which underwent different chemical treatments, such as buffered chemical polishing (BCP) or electropolishing (EP), heat treatments such as post-purification or with different crystallographic properties, such as cavities made of single crystal niobium.

\section{General results from rf tests on $\mathrm{Nb}$ cavities}

Polycrystalline niobium surfaces treated by $\mathrm{BCP}$ are characterized by grain sizes of the order of $50 \mu \mathrm{m}$ and roughnesses of the order of 5-10 $\mu \mathrm{m}$ [5], after a typical removal of $\sim$ $100 \mu \mathrm{m}$. It has been observed that baking only pushes the onset field of the $Q$-drop to higher values but the losses are still present. This can be seen, for example, in Fig. 1a which shows the rf test results for a $2.25 \mathrm{GHz}$ single cell cavity [6] before and after baking at $120^{\circ} \mathrm{C}$ for $48 \mathrm{~h}$ and Fig. $1 \mathrm{~b}$ which shows similar results on a $1.3 \mathrm{GHz}$ single cell cavity measured before and after baking at $100{ }^{\circ} \mathrm{C}$ for $48 \mathrm{~h}[7]$.

A post-purification treatment is commonly applied to improve the thermal conductivity of the niobium by heating the cavity in a vacuum furnace to temperatures greater than $1000{ }^{\circ} \mathrm{C}$ for many hours, in the presence of a solid state getter (Ti, Yt, Pd) [8]. As a result of this process, the niobium recrystallizes in millimiter-size grains. Subsequent etching by $\mathrm{BCP}$ still provides surfaces with $5-10 \mu \mathrm{m}$ roughness and sharp edges at grain boundaries. Measurements of cavities which were treated in such a way show a significant reduction of the $Q$-drop and they are limited by a thermal quench, possibly due to defects in the material. This is shown, for example, in Fig. 2a for a $1.47 \mathrm{GHz}$ single cell baked at $120^{\circ} \mathrm{C}$ for $48 \mathrm{~h}[9]$ and in Fig. $2 \mathrm{~b}$ for a $1.3 \mathrm{GHz}$ single cell baked at 
$110^{\circ} \mathrm{C}$ for $60 \mathrm{~h} \mathrm{[10].} \mathrm{It} \mathrm{is} \mathrm{worthwhile} \mathrm{to} \mathrm{mention} \mathrm{that} \mathrm{no} \mathrm{significant} \mathrm{differences} \mathrm{in} \mathrm{the}$ cavity results were observed for $\mathrm{BCP}$ with a mixture of the acids $\mathrm{HF}, \mathrm{HNO}_{3}$ and $\mathrm{H}_{3} \mathrm{PO}_{4}$ in $1: 1: 1$ or $1: 1: 2$ ratios.

Visentin [11] measured a post-purified cavity after baking in air, rather than under ultrahigh vacuum conditions ("in-situ"), at $110^{\circ} \mathrm{C}$ for $60 \mathrm{~h}$ and obtained some improvement of the $Q$-drop (Fig. 3b). A similar test performed at Jefferson Lab on a cavity which was not post-purified showed no improvement after baking at $120{ }^{\circ} \mathrm{C}$ for $48 \mathrm{~h}$ (Fig. 3a) [12]. Also, the increase of residual resistance, which is often seen after baking, was much higher than obtained with "in-situ" baking in both cases. This might suggest that the parameters of the "air" baking need to be optimized.

Polycrystalline cavities treated by EP are smoother (roughness of about 2-5 $\mu \mathrm{m}$ [5]) than BCP-treated ones and rf test results show that the $Q$-drop is greatly reduced by baking, as shown for example in Fig. 4a, on a $1.3 \mathrm{GHz}$ single cell baked at $110^{\circ} \mathrm{C}$ for $30 \mathrm{~h}$ [13]. Similar results are achieved on cavities which had been post-purified, as shown in Fig. $4 \mathrm{~b}$ on a $1.3 \mathrm{GHz} 9$-cell cavity baked at $120^{\circ} \mathrm{C}$ for $48 \mathrm{~h}[14]$.

Recently, two cavities made of single crystal niobium were built at Jefferson Lab [6] and mirror-like surfaces (roughness of less than $1 \mu \mathrm{m}$ [15]) were obtained. Figure 5 shows the results from the rf tests of the cavity with "High Gradient" shape at $2.25 \mathrm{GHz}$ (a) and of the one with "Low Loss" shape at $2.36 \mathrm{GHz}(\mathrm{b})$, where a significant reduction of the $Q$ drop is obtained after baking at $120^{\circ} \mathrm{C}$ for $48 \mathrm{~h}$ and $24 \mathrm{~h}$ respectively.

Figure 6 shows the statistic on the onset $B_{p}$-field of the $Q$-drop measured on $1.47 \mathrm{GHz}$ single cells built and tested at Jefferson Lab [12]: the average value before postpurification is $87 \pm 11 \mathrm{mT}$, increasing to $104 \pm 11 \mathrm{mT}$ after post-purification. Even higher 
onsets were achieved on large grain (centimeter-size) cavities and on one electropolished cavity.

A comparison between measurements of the temperature dependence of the low-field surface impedance and the BCS theory on a niobium cavity showed that the mean free path of the normal electrons $(l)$, in a depth of the order of the rf penetration depth $(\sim 40$ $\mathrm{nm}$ ), is significantly reduced by baking at $120{ }^{\circ} \mathrm{C}$ for $48 \mathrm{~h}$ [9], so that the physics of the niobium surface changes from the so-called "clean limit" $\left(l>>\xi_{0}\right.$, where $\xi_{0}$ is the coherence length) to the "dirty limit" $\left(l \approx \xi_{0}\right)$. This effect, combined with a slight increase $(\cong 2-5 \%)$ of the ratio $\Delta(0 \mathrm{~K}) / k T_{c}$, causes a reduction of the BCS surface resistance up to about $50 \%$, improving the quality factor of the cavity at low field.

\section{General results from surface measurements on Nb samples}

In parallel to rf tests on cavities, many different studies have been done on $\mathrm{Nb}$ samples to investigate the effect of baking. Since the rf current in a cavity flows in a depth of only about $40 \mathrm{~nm}$, it is useful to investigate changes in the niobium surface due to different treatments. It was commonly found $[16,17,18]$ that low-temperature baking causes a partial decomposition of the natural oxide layer $\left(\mathrm{Nb}_{2} \mathrm{O}_{5}\right)$ on the $\mathrm{Nb}$ surface which converts into sub-oxides $\left(\mathrm{NbO}, \mathrm{NbO}_{2}, \mathrm{Nb}_{2} \mathrm{O}\right)$ and provides oxygen which can diffuse deeper into the metal. It was also found that oxygen segregates near the metal/oxide interface and concentrations up to 10 at. \% had been measured, in about $1 \mathrm{~nm}$ layer, on a single crystal sample treated by BCP [19]. Besides oxygen, the other most important impurity in niobium is hydrogen. Recent results on hydrogen distribution near the surface by nuclear reaction analysis show that hydrogen is displaced from the metal/oxide 
interface by baking [9]. In general, the relation between hydrogen and the baking effect is not clear yet and requires further investigations.

Measurements of the susceptibility on Nb samples treated by EP and BCP showed an increase of the ratio between the surface critical field $\left(B_{c 3}\right)$ and the upper critical field $\left(B_{c 2}\right)$ by baking [20] and this was consistent with the presence of an impurity layer of thickness $d<<\xi_{0}$ and with a reduction of $l$. Higher baking temperatures or longer duration would reduce $l$ and increase $d$.

Magneto-optical measurements on $\mathrm{Nb}$ samples treated by $\mathrm{BCP}$ have been done recently [21] to investigate the possibility of flux penetration at grain boundaries. Indeed, a sample with millimiter-size grains, grown by post-purification, showed evidence of flux penetrating across grain boundaries at a field of the order of $B_{c l}$ (lower critical field), as shown in Fig. 7.

\section{Investigations of the origin of the Q-drop}

One of the models to explain the $Q$-drop and the baking effect, proposed by Halbritter and explained in details in Ref. [22], deals with enhanced interface losses between the natural pentoxide layer and the niobium metal. The model considers the $Q$-drop as being triggered by a high surface electric field and by the presence of a high density of localized states in the oxide layer. The surface resistance is predicted to increase exponentially with the rf field and this dependence well describes the experimental data, as for example, in Ref. [9].

All other models for the $Q$-drop, reviewed in [23], consider a high magnetic field as the cause for the losses. Several experiments were conducted in different laboratories to 
investigate the origin of the $Q$-drop. At Jefferson Lab, the $Q_{0}$ as a function of $B_{p}$ was measured in the usual $\mathrm{TM}_{010}($ at $1.47 \mathrm{GHz})$ and in the $\mathrm{TE}_{011}($ at $2.82 \mathrm{GHz}$ ) in the same single cell cavity [24]. After post-purification, the $Q$-drop was clearly identified in both modes and was strongly reduced by baking, as shown for example in Fig. 8. Since in the $\mathrm{TE}_{011}$ mode there is no surface electric field, the $Q$-drop appears to be caused by a high magnetic field. Similar conclusions could be drawn from the results of the rf tests of a two-cell cavity designed to have significantly different distributions of the surface electric and magnetic fields in the pass-band modes of the $\mathrm{TM}_{010}$ family [25].

At Saclay, a $1.3 \mathrm{GHz}$ single cell cavity was rinsed with HF after baking, but in the subsequent $\mathrm{rf}$ test the $Q$-drop was not restored [26]. If the benefit of baking effect would be due to a thinning of the oxide layer, as predicted by Halbritter's model, the growth of a fresh oxide, as after the HF treatment, should restore the $Q$-drop.

In addition, temperature maps of the cavity surface measured in different laboratories [12, $27,28]$ agree in showing heat originating in the equator area of the cavities, where the magnetic field is highest in the $\mathrm{TM}_{010}$ mode, causing the increase of the surface resistance and therefore a drop of $Q_{0}$. The losses do not appear to be uniformly distributed along the cavity equator, but there are a number of "hot spots" which are expanding for increasing rf field. This is shown for example in Fig. 9 [12].

In order to investigate up to what depth the changes in the niobium surface due to baking allow a reduction of the $Q$-drop, an experiment was done at Cornell University where a 1.3 GHz single cell cavity was anodized after baking, converting about $20 \mathrm{~nm}$ of niobium into pentoxide [7]. As a result, the $Q$-drop was restored as prior to baking. 


\section{Discussion on the Q-drop and the baking effect}

The experimental results on cavities rf tests presented in Sec. 2 indicate that the onset field of the $Q$-drop is increased in cavities with smoother surfaces and/or larger grains. Baking seems to be more effective in reducing the $Q$-drop on smoother surfaces, such as by EP, or on cavities with large grains. The results discussed in Sec. 4 also suggest that a high magnetic field is responsible for the anomalous losses and that the surface oxide layer does not contribute appreciably to the $Q$-drop and its reduction by baking. The experimental results on $\mathrm{Nb}$ samples (Sec. 3) indicate that baking causes oxygen to re-distribute in the proximity of the niobium surface by diffusion and that flux penetration can occur at grain boundaries at fields close to $B_{c l}$.

It is known that interstitial oxygen in niobium lowers the critical temperature, $T_{c}$, and increases the Ginzburg-Landau parameter, $\kappa$ [29]. As a consequence, the value of the lower critical field of $\mathrm{Nb}$ can be reduced by the presence of interstitial oxygen. In particular, a reduction of $B_{c l}$ by about a factor of two (corresponding to the onset field for the $Q$-drop) can be achieved with an oxygen concentration of about 0.56 at. $\%[29,30]$. This value is consistent with the results from surface analysis on $\mathrm{Nb}$ samples, where significantly higher concentrations were obtained in a $1 \mathrm{~nm}$ layer at the metal/oxide interface. Flux penetration in a superconductor can be inhibited above $B_{c l}$ by a surface barrier, as shown by Bean and Livingston [31], but such barrier can be strongly reduced in the presence of a rough surface [32] or of an impurity layer [33].

In summary, we can suggest a model, similar to one proposed by Safa in Ref. [34], where the onset of the $Q$-drop corresponds to the onset field for fluxoids penetrating the niobium surface. The value of this onset field is determined by the surface barrier, being 
reduced by the presence of a high oxygen concentration near the metal/oxide interface and by rough surfaces. This could explain the lower value of the onset field of the $Q$-drop for BCP-treated cavities versus EP-treated or single/large grain cavities. Baking at an optimum temperature and time would allow oxygen to dilute over the penetration depth, with concentration values which would be more homogeneously distributed. A possible way to quantitatively evaluate the effect of baking is to solve the diffusion equation for oxygen as a function of baking time and temperature. In the past, two extreme approaches were taken when establishing the boundary conditions for the oxygen concentration at the metal oxide interface: in one case the oxide layer is viewed as a constant source of oxygen during bake-out [35], while in another case the contribution of the oxide layer was ignored [34]. As discussed in Sect. 3, surface analysis measurements show that oxide acts as a source of oxygen due to partial decomposition of the $\mathrm{Nb}_{2} \mathrm{O}_{5}$ layer. We implemented this effect in the solution of Fick's equation for the oxygen concentration, $c(x, t, T)$, obtaining the following expression [36]:

$$
c(x, t, T)=\frac{u_{0}}{\sqrt{\pi D(T)}} \int_{0}^{t} \frac{k(T) e^{-k(T) s}}{\sqrt{t-s}} e^{-\frac{x^{2}}{4 D(T)(t-s)}} d s+\frac{v_{0}}{\sqrt{\pi D(T) t}} e^{-\frac{x^{2}}{4 D(T) t}}
$$

where $D(T)$ is the diffusion constant of oxygen in niobium, $k(T)$ is the oxide reduction rate, $u_{0}$ and $v_{0}$ are the amount of oxygen per unit area initially present in the oxide and as interstitial impurity in niobium respectively. $x$ is the depth from the metal/oxide interface, at $x=0$.

A plot of $c(x, t, T)$ for $x=0$ and $t=48 \mathrm{~h}$ as a function of the baking temperature is shown in Fig. 10 [36], indicating that the oxygen concentration at the metal/oxide interface is 
reduced by baking in the temperature range between $120-150^{\circ} \mathrm{C}$, consistently with the proposed model.

Fluxoids in niobium would yield additional losses, mainly by Joule heating of their normal-conducting core, reducing the cavity quality factor. Rabinowitz estimated the power dissipation due to a single isolated fluxoid in an rf field [37] and obtained an exponential increase of the surface resistance, once the fluxoid enters in the niobium, which well describes the experimental data. Rabinowitz also predicted that for the case of fluxoids with negligible viscous damping and negligible pinning the field of flux penetration increases linearly with the rf frequency [38]. The $Q$-drop onset field measured on cavities at different frequencies, between 700 and $2.82 \mathrm{GHz}$, seems to be consistent with this prediction, as shown in Fig. 11, although it should be mentioned that the $Q$-drop hasn't been found on low-beta, non-elliptical cavities $(115-354 \mathrm{MHz})$ up to $B_{p} \approx 80 \mathrm{mT}[39]$.

\section{Summary}

The main limitation towards achieving the rf critical field in niobium superconducting cavities is represented by the so-called $Q$-drop, which consists in a sharp decrease of the cavity quality factor starting at $B_{p} \approx 100 \mathrm{mT}$, in absence of field emission. A low temperature "in-situ" baking of the cavity allows a reduction of these anomalous losses. Cavity test results show that the onset field of the $Q$-drop and the baking effect depend on the density of grain boundaries and on the smoothness of the surface. Cavities with smoother surfaces and reduced density of grain boundaries consistently achieve higher accelerating gradients, with $Q$-drop starting at higher fields and being strongly reduced 
by baking. Cavity test results also show that the $Q$-drop is caused by a high magnetic, rather than electric field, and that the presence of an oxide layer on the niobium surface does not seem to introduce high-field losses.

Measurements on $\mathrm{Nb}$ samples support the hypothesis of oxygen diffusing away from the metal/oxide interface during bake-out and that flux penetration may occur at grain boundaries at fields close to $B_{c l}$. All these observations can be consistently related by a model which considers flux penetration at a reduced $B_{c l}$ as the main cause for the $Q$-drop. The reduction of $B_{c l}$ is due to the presence of a thin layer with a high oxygen concentration, which is being reduced by baking at optimum time/temperature. With an improved model of oxygen diffusion, which includes the contribution of the oxide, we could verify that, for a fixed time, there exists a temperature range for baking which allows a reduction of the oxygen concentration near the surface.

Besides this model, other effects such as a geometric field enhancement due to rough surfaces might contribute to the $Q$-drop, so that it may be difficult to find a single explanation for all the cavity test results.

Further validation of the proposed flux penetration model would require some additional measurements and theoretical work. For example, it would be useful to have quantitative measurements of the oxygen content as a function of depth, before and after baking and to conduct experiments on cavities to proof the presence of fluxoids during the $Q$-drop. From the theoretical point of view, it should also be clarified whether the time-scale of the $r f$ is compatible with the nucleation time for fluxoids and whether the losses associated with them are large enough to describe the observed increase of the surface resistance. 
Another theoretical challenge is to determine whether the change from clean to dirty limit of the niobium surface after baking has significant implications on the surface resistance at high field. For example, Gurevich calculated an expression of the high-field surface resistance in the clean limit which shows an exponential increase due to pair-breaking effect by the rf field [40].

In conclusion, the understanding of the high-field $Q$-drop and of the baking effect is of great importance to reliably produce superconducting niobium cavities with accelerating gradients above $30 \mathrm{MV} / \mathrm{m}$, as required for projects such as the International Linear Collider [41].

We would like to acknowledge P. Kneisel, G. R. Myneni, J. Halbritter, J. Delayen, B. Visentin, A. Gurevich and H. Padamsee for many useful discussions. This work was supported by the U.S. DOE Contract No DE-AC05-84ER40150 Modification No. M175, under which the Southeastern Universities Research Association (SURA) operates the Thomas Jefferson National Accelerator Facility. 


\section{References}

[1] P. Kneisel, K. Saito, and R. Parodi, Proceedings of the $8^{\text {th }}$ Workshop on RF Superconductivity, Abano Terme, Italy, 1997, p. 463.

[2] E. Kako et al.,Proceedings of the $8^{\text {th }}$ Workshop on RF Superconductivity, Abano Terme, Italy, 1997, p. 491.

[3] P. Kneisel, Proceedings of the $9^{\text {th }}$ Workshop on RF Superconductivity, Santa Fe, NM, 1999, p. 328.

[4] B. Visentin, J. P. Charrier, and B. Coadou, Proceedings of the 1998 European Particle Accelerator Conference, Stockholm, Sweden, 1998, p. 1885.

[5] R. L. Geng, J. Knobloch, and H. Padamsee, Proceedings of the $9^{\text {th }}$ Workshop on RF Superconductivity, Santa Fe, NM, 1999, p. 238.

[6] P. Kneisel, G. Ciovati, G. R. Myneni, T. Carneiro, and J. Sekutowicz, Proceedings of the 2005 Particle Accelerator Conference, Knoxville, TN, 2005, TPPT076.

[7] G. Eremeev et al., Proceedings of the Pushing the Limits of RF Superconductivity Workshop, Argonne, IL, 2004, p. 291.

[8] H. Safa et al., Proceedings of the $7^{\text {th }}$ Workshop on RF Superconductivity, Gif sur Yvette, France, 1995, p. 649.

[9] G. Ciovati, J. Appl. Phys. 96 (2004) 1591.

[10] B. Visentin et al., Proceedings of the 2002 European Particle Accelerator Conference, Paris, France, p. 2292

[11] B. Visentin et al., Proceedings of the $11^{\text {th }}$ Workshop on RF Superconductivity, Travemünde, Germany, 2003, MoP19.

[12] G. Ciovati, Ph.D. Thesis, Old Dominion University, Norfolk, 2005. 
[13] B. Visentin et al., Proceedings of the $11^{\text {th }}$ Workshop on RF Superconductivity, Travemünde, Germany, 2003, TuO01.

[14] L. Lilje, Proceedings of the 2004 European Particle Accelerator Conference, Lucerne, Switzerland, 2004, p. 129.

[15] A. Wu, private communication.

[16] Q. Ma and R. Rosenberg, Proceedings of the $10^{\text {th }}$ Workshop on RF Superconductivity, Tsukuba, Japan, 2001, p. 368.

[17] A. Daccà, G. Gemme, L. Mattera, and R. Parodi, Appl. Surf. Sci 126 (1998) 219

[18] K. Kowalski et al., Proceedings of the $11^{\text {th }}$ Workshop on RF Superconductivity, Travemünde, Germany, 2003, ThP09.

[19] I. Arfaoui, C. Guillot, J. Cousty, and C. Antoine, J. Appl. Phys. 91 (2002) 9319.

[20] S. Casalbuoni, L. von Sawilski, and J. Kotzler, Proceedings of the $11^{\text {th }}$ Workshop on RF Superconductivity, Travemünde, Germany, 2003, WeO13.

[21] P. Lee et al., Proceedings of the Pushing the Limits of RF Superconductivity Workshop, Argonne, IL, 2004, p. 84.

[22] J. Halbritter, Proceedings of the $10^{\text {th }}$ Workshop on RF Superconductivity, Tsukuba, Japan, 2001, p 291.

[23] B. Visentin, Proceedings of the $11^{\text {th }}$ Workshop on RF Superconductivity, Travemünde, Germany, 2003, TuO01.

[24] G. Ciovati and P. Kneisel, Proceedings of the Pushing the Limits of RF Superconductivity Workshop, Argonne, IL, 2004, p. 74.

[25] G. Ciovati, P. Kneisel, J. Sekutowicz, and W. Singer, see Ref. [6], TPPT072. 
[26] B. Visentin, Proceedings of the Pushing the Limits of RF Superconductivity Workshop, Argonne, IL, 2004, p. 94.

[27] L. Lilje et al., Proceedings of the $9^{\text {th }}$ Workshop on RF Superconductivity, Santa Fe, NM, 1999, p. 74 and private communication.

[28] G. Eremeev, H. Padamsee, M. Liepe, and R. Roy, see Ref. [11], MoP18.

[29] C. C. Koch, J. O. Scarbrough, and D. M. Kroeger, Phys. Rev. B 9 (1974) 888.

[30] J. R. Waldram, Superconductivity of Metals and Cuprates (Institute of Physics Publishing, Bristol, 1996), Chap. 5, p.75.

[31] C. Bean and J. D. Livingston, Phys. Rev. Lett. 12 (1964) 14.

[32] R. D. Blois and W. de Sorbo, Phys. Rev. Lett. 12 (1964) 499.

[33] A. Gurevich and V. T. Kovachev, Phys. Stat. Sol. B 145 (1988) K47.

[34] H. Safa, Proceedings of the $10^{\text {th }}$ Workshop on RF Superconductivity, Tsukuba, Japan, 2001, p. 279.

[35] C. Benvenuti, S. Calatroni, and V. Ruzinov, Proceedings of the $10^{\text {th }}$ Workshop on RF Superconductivity, Tsukuba, Japan, 2001, p. 441.

[36] G. Ciovati, submitted to Appl. Phys. Lett.

[37] M. Rabinowitz, J. of. Appl. Phys. 42 (1971) 88.

[38] M. Rabinowitz, Appl. Phys. Lett. 19 (1971) 73.

[39] M. P. Kelly, J. D. Fuerst, M. Kedzie, K. W. Shepard, Z. A. Conway, Proceedings of the Pushing the Limits of RF Superconductivity Workshop, Argonne, IL, 2004, p. 278. [40] A. Gurevich, this conference, TuA01. [41] http://www.interactions.org/linearcollider 


\section{Figure legends}

1. $Q_{0}$ vs. $E_{p}$ measured on a $2.25 \mathrm{GHz}$ single cell cavity [6] before and after baking at 120

${ }^{\circ} \mathrm{C}$ for $48 \mathrm{~h}$ (a) and on a $1.3 \mathrm{GHz}$ single cell cavity [7] before and after baking at $100{ }^{\circ} \mathrm{C}$ for $48 \mathrm{~h}(\mathrm{~b})$, both made from fine grain RRR $>200 \mathrm{Nb}$ and treated with BCP.
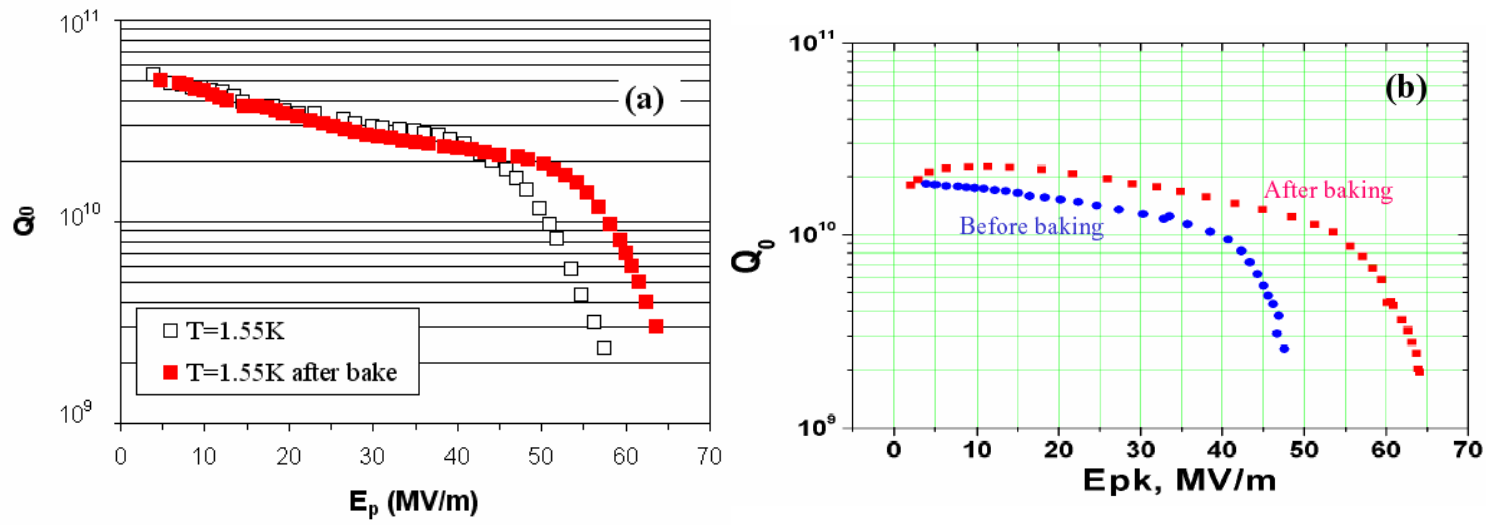

2. $Q_{0}$ vs. $E_{\text {acc }}$ measured on a $1.47 \mathrm{GHz}$ single cell cavity [9] before and after baking at $120{ }^{\circ} \mathrm{C}$ for $48 \mathrm{~h}$ (a) and on a $1.3 \mathrm{GHz}$ single cell cavity [10] before and after baking at $100{ }^{\circ} \mathrm{C}$ for $48 \mathrm{~h}(\mathrm{~b})$, both cavities were post-purified at $1400{ }^{\circ} \mathrm{C}$ and treated with $\mathrm{BCP}$.
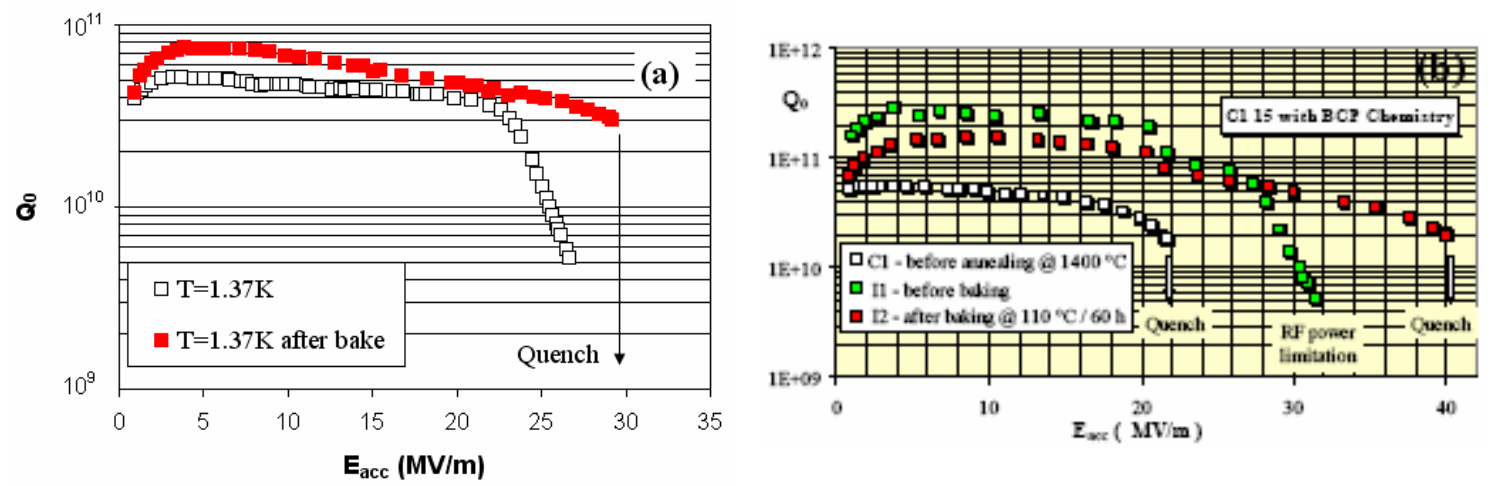

3. $Q_{0}$ vs. $E_{\text {acc }}$ measured on a $1.47 \mathrm{GHz}$ single cell cavity [12] before and after baking at $120{ }^{\circ} \mathrm{C}$ for $48 \mathrm{~h}$ (a) and on a $1.3 \mathrm{GHz}$ single cell cavity [11] before and after baking at $110^{\circ} \mathrm{C}$ for $60 \mathrm{~h}(\mathrm{~b})$. Both cavities were baked at atmospheric pressure, rather than under 
ultra-high vacuum and were treated with BCP. The cavity in (a) was not post-purified while the cavity in (b) was.
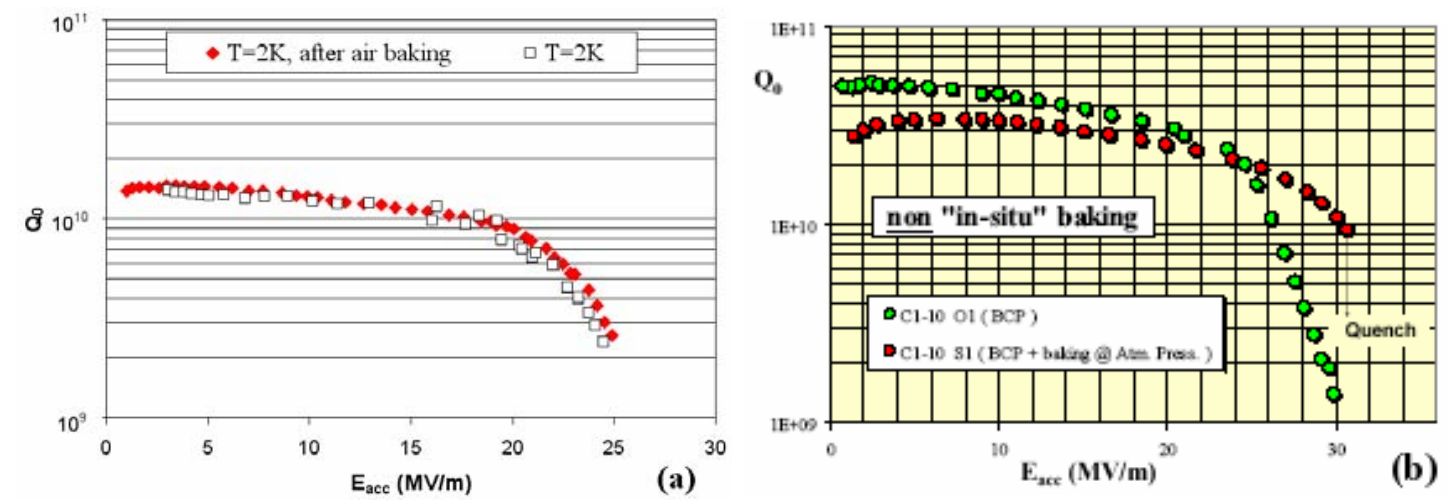

4. $Q_{0}$ vs. $E_{a c c}$ measured on a post-purified $1.3 \mathrm{GHz}$ single cell cavity [12] before and after baking at $110^{\circ} \mathrm{C}$ for $30 \mathrm{~h}$ (a) and on a $1.3 \mathrm{GHz}$ 9-cell cavity [11] before and after baking at $120^{\circ} \mathrm{C}$ for $48 \mathrm{~h}$ (b). Both cavities were treated with EP.
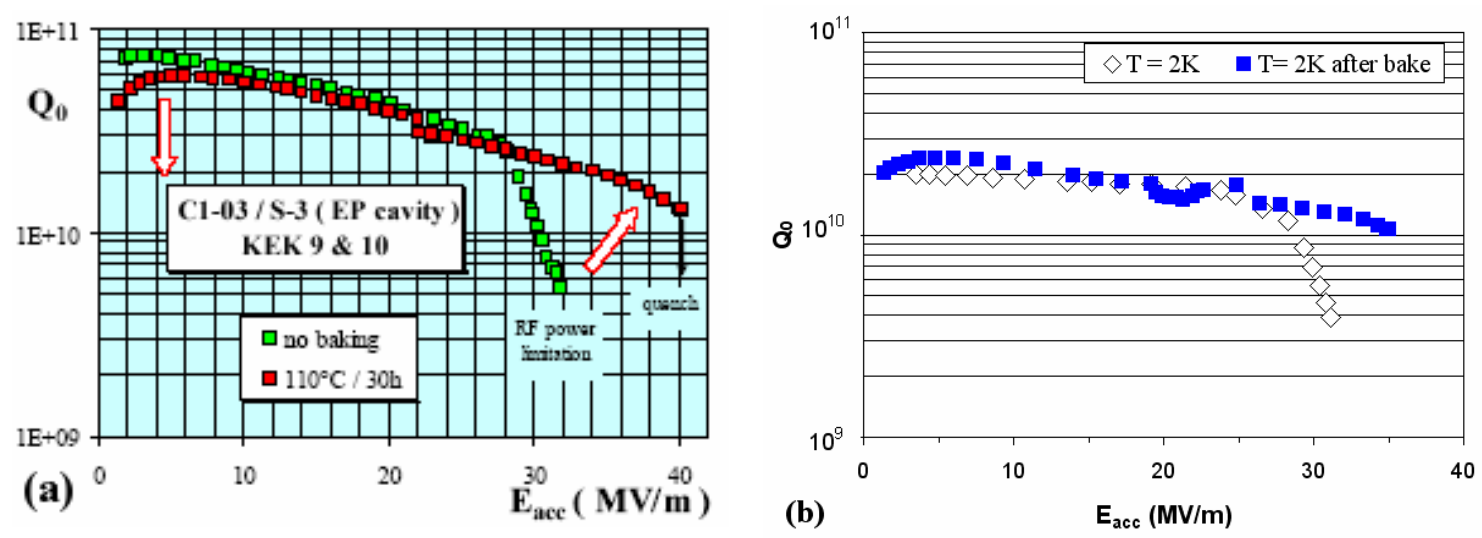

5. $Q_{0}$ vs. $E_{a c c}$ measured on a post-purified $2.25 \mathrm{GHz}$ single cell cavity (a) and on a 2.36

$\mathrm{GHz}$ single cell cavity (b) before and after baking at $120^{\circ} \mathrm{C}$ for $48 \mathrm{~h}$ and $24 \mathrm{~h}$

respectively [6]. Both cavities were made from single crystal $\mathrm{Nb}$ disks and were treated with BCP. 

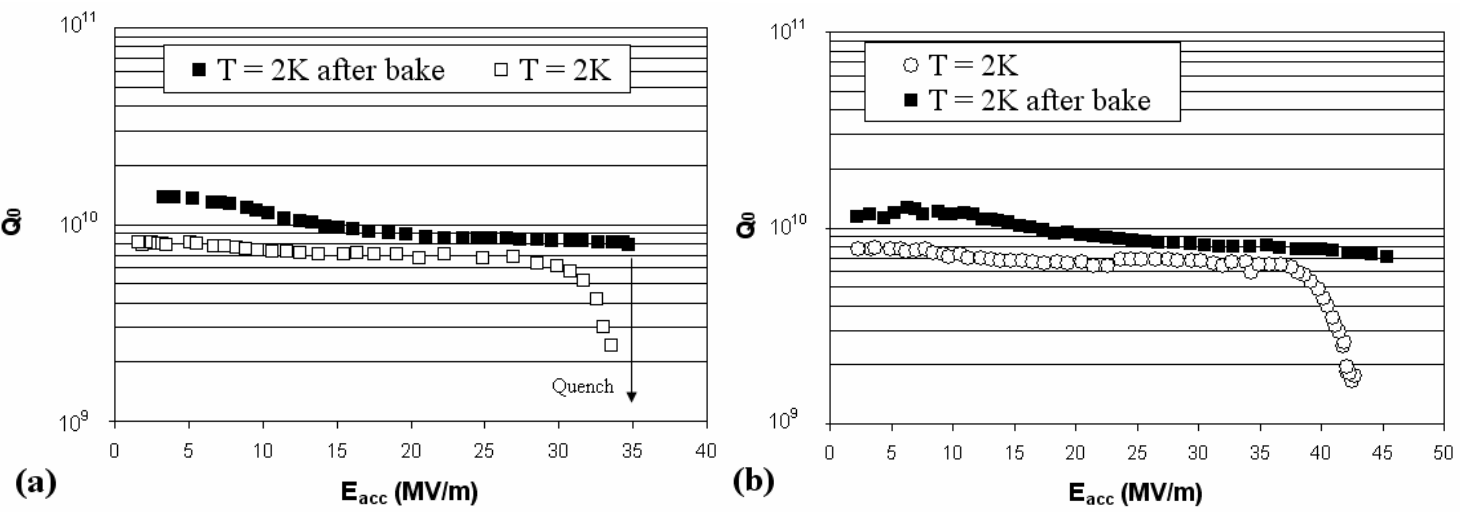

6. Distribution of the $Q$-drop onset field measured on $1.47 \mathrm{GHz}$ single cell cavities at Jefferson Lab with different chemical and heat treatments [12].

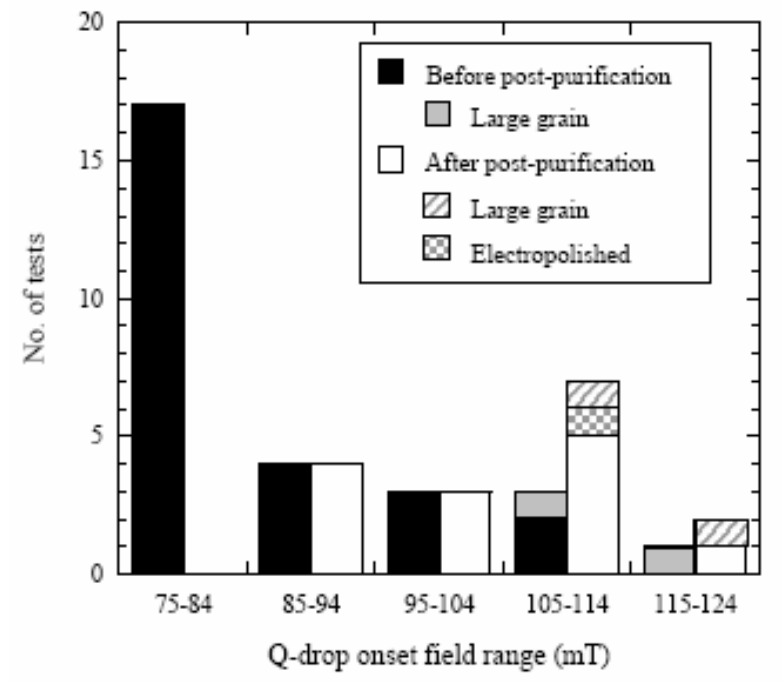

7. Measurements of flux penetration in a post-purified $\mathrm{Nb}$ sample treated by BCP. Left: optical image, Right: magneto-optical image at $7 \mathrm{~K}$ in an external field of $57 \mathrm{mT}$ [21]. 

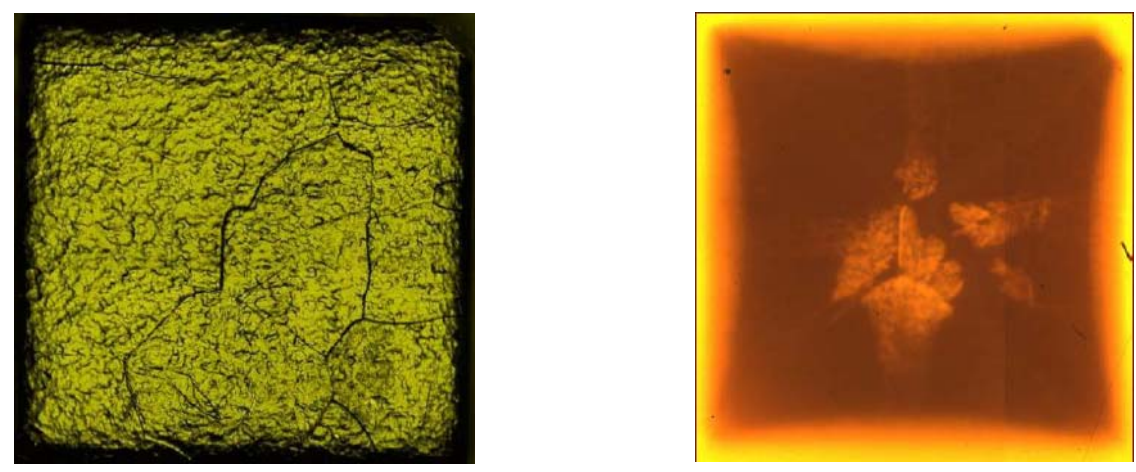

8. $Q_{0}$ vs. $B_{p}$ measured in the $\mathrm{TM}_{010}$ mode $(1.47 \mathrm{GHz})$ and in the $\mathrm{TE}_{011}$ mode $(2.82 \mathrm{GHz})$ of a post-purified single cell cavity before and after baking at $120{ }^{\circ} \mathrm{C}$ for $30 \mathrm{~h} \mathrm{[24]}$.

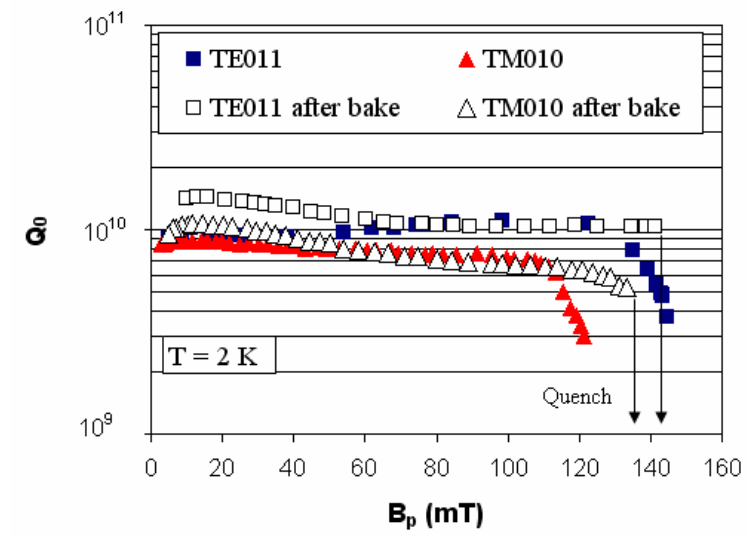

9. Temperature map measured at $2 \mathrm{~K}$ on a fine-grain single cell, showing hot-spots in the equator region causing the $Q$-drop [12]. 


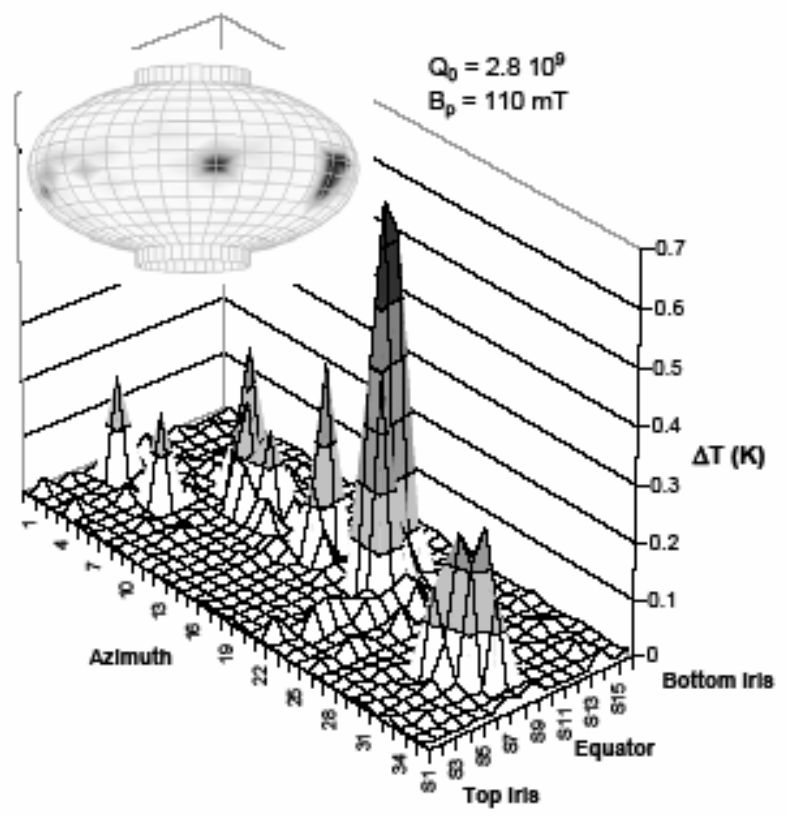

10. Oxygen concentration at the metal/oxide interface as a function of the baking temperature calculated with Eq. (1) for a baking duration of $48 \mathrm{~h}$ [36]. The dashed line represents the contribution for the decomposition of the oxide layer, while the dashdotted line represents the interstitial oxygen initially present at the metal/oxide interface.

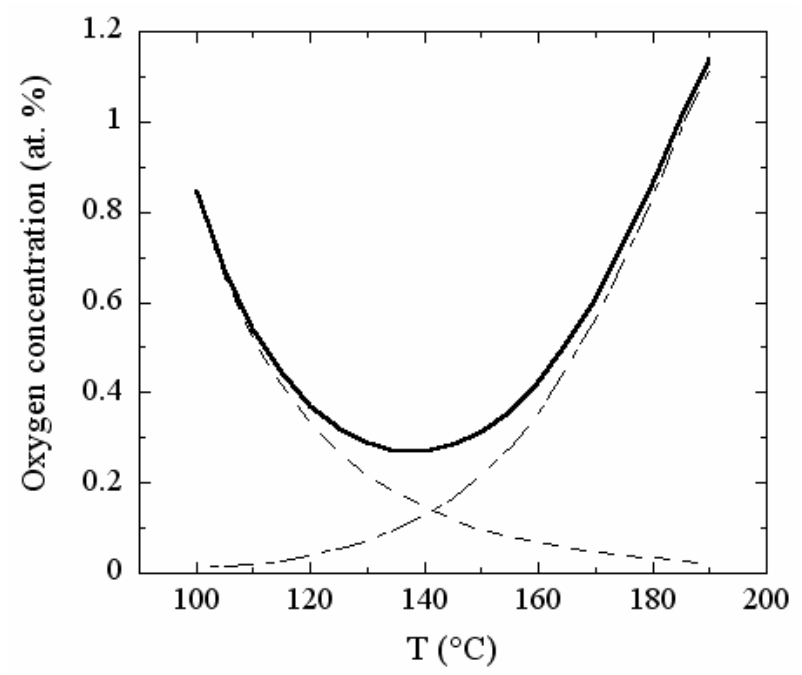

11. $Q$-drop onset field measured at different frequencies [12]. The data point at $2.82 \mathrm{GHz}$ was measured in the $\mathrm{TE}_{011}$ mode. 


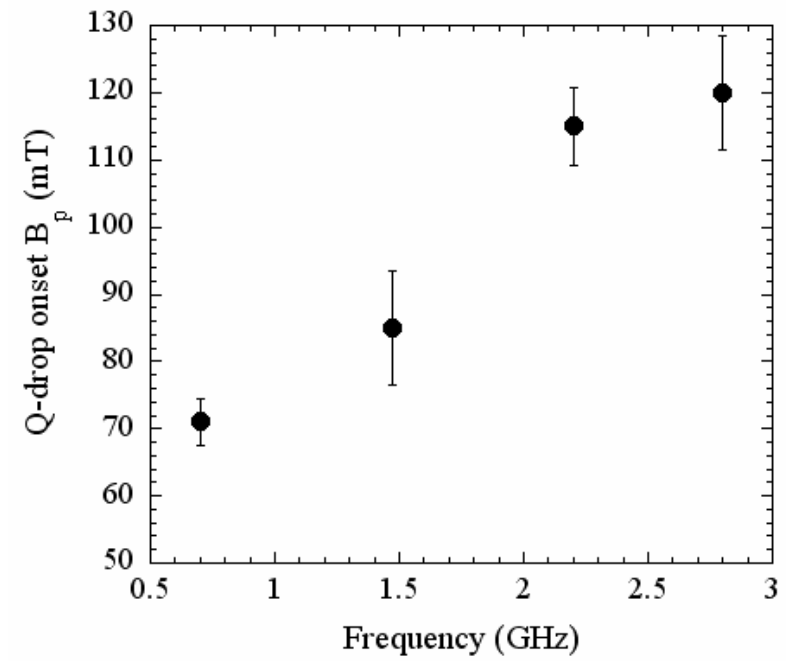

